\title{
A Meta-Analysis on the Effect and Safety of LCZ696 in the Treatment of Hypertension
}

\author{
Li Zheng $\mathbb{D}^{1}{ }^{1}$ Binbin Xia, ${ }^{2}$ Xuqian Zhang, ${ }^{3}$ and Yan Zhao $\mathbb{D}^{1}$ \\ ${ }^{1}$ Department of Pharmacy, China Aerospace Science \& Industry Corporation 731 Hospital, Beijing 100074, China \\ ${ }^{2}$ Department of Pharmacy, Luhe Hospital, Beijing 101149, China \\ ${ }^{3}$ Department of Gastroenterology and Hepatology, China Aerospace Science \& Industry Corporation 731 Hospital, \\ Beijing 100074, China \\ Correspondence should be addressed to Yan Zhao; 13520339253@163.com
}

Received 27 September 2020; Revised 27 January 2021; Accepted 3 March 2021; Published 22 March 2021

Academic Editor: Francesco Fedele

Copyright (c) 2021 Li Zheng et al. This is an open access article distributed under the Creative Commons Attribution License, which permits unrestricted use, distribution, and reproduction in any medium, provided the original work is properly cited.

\begin{abstract}
Objectives. To systematically evaluate the differences in effect and safety of LCZ696 and angiotensin receptor blockers (ARBs) in the treatment of hypertension. Methods. We searched PubMed, Cochrane, Web of Science, and Ovid, collected randomized controlled trials (RCTs) about the effect and safety of LCZ696 and ARBs in hypertensive patients, extracted relevant data and evaluated the quality of the included literature according to the RCT quality evaluation standard recommended by Cochrane Reviewer's Handbook, using RevMan 5.3, and performed meta-analysis. Results. Eight RCTs studies were included, with a total of 4313 patients. Compared with ARBs, LCZ696 can better reduce systolic blood pressure (msSBP) (WMD $-4.29 \mathrm{mmHg}$; $95 \%$ CI: -5.37 to $-3.21 ; P<0.001$ ), diastolic blood pressure (msDBP) (WMD $-1.87 \mathrm{mmHg}$; $95 \% \mathrm{CI}:-2.38$ to $-1.36 ; P<0.01$ ), ambulatory systolic blood pressure (maSBP) (WMD $-3.37 \mathrm{mmHg}$; 95\% CI:-4.26 to $-2.47 ; P<0.01$ ), and ambulatory diastolic blood pressure (maDBP) (WMD $-1.47 \mathrm{mmHg}$; 95\% CI: -1.97 to $-0.97 ; P<0.01$ ). In terms of safety, LCZ696 is basically the same as ARBs, but LCZ696 is more likely to cause cough than ARBs (OR $=2.38$; $95 \%$ CI: 1.27 to 4.47; $P<0.01$ ). Conclusion. LCZ696 can significantly reduce the blood pressure level of patients with hypertension, but it is necessary to pay attention to whether the patient will experience coughing after taking the drug.
\end{abstract}

\section{Introduction}

Hypertension is a chronic condition that contributes to worldwide morbidity and mortality more than any other risk factor. As reported, the number of patients with hypertension was estimated to be $31.1 \%$ among adults in 2010 [1]. Hypertension is the most common cardiovascular leading risk factor and underlies heart failure (HF), coronary artery disease, stroke, and chronic kidney disease. Hypertensive heart disease includes the development of diastolic dysfunction, left ventricular hypertrophy, and HF with preserved and reduced ejection fraction [2]. Therefore, the optimal treatment of hypertension can prevent the development of HF.
Blood pressure can be controlled by changing diet and lifestyle, but drug treatment is used when these methods are not effective. Commonly prescribed antihypertensive drugs include thiazide-diuretics, $\beta$-blockers, calcium channel blockers, angiotensin converting enzyme (ACE) inhibitors, and angiotensin II receptor blockers (ARB) [3]. However, lots of treated patients still cannot effectively control blood pressure level, and we need to keep looking for better antihypertensive agents to achieve $\mathrm{BP}$ goals and reduce cardiovascular events.

Various studies have revealed the potential of LCZ696 as an antihypertensive drug [4-6]. LCZ696 provides highly selective inhibition of neprilysin and blockade of the angiotensin receptor. It plays an important role in improving 
$\mathrm{HF}$, reducing ejection fraction, and controlling blood pressure [7, 8]. Here, we searched studies and performed a meta-analysis on available randomized clinical (RCT) trials to research the effect and safety of LCZ696 in hypertension patients.

\section{Methods}

Our search strategy is illustrated in the Preferred Reporting Items for Systematic Reviews and Meta-analyses (PRISMA) [9] reporting guideline flowchart in Figure 1.

2.1. Search Strategy. All data were obtained by actively searching PubMed, Cochrane, Web of Science, and Ovid up to Sep 1, 2020. Keywords and Medical Subject Headings (MeSH) included the following: "LCZ696," "sacubitril/valsartan," “AHU377," “angiotensin receptor-neprilysin inhibitor (ARNi)," and "hypertension."

2.2. Inclusion Criteria. Studies meeting the following criteria were selected:

(1) The trial was a RCT with complete data

(2) Patients with clinical diagnosis of hypertension

(3) LCZ696 was used in the experimental group (any dose)

(4) ARBs were used in the control group

(5) There were adverse events (AEs) in the outcome indicators

2.3. Exclusion Criteria. Studies that used only self-report or informant report as an outcome were excluded. We excluded animal experimental studies as well as case reports from the meta-analysis. In the same way, all studies that are not written in English, non-RCT studies, duplicate studies, single-arm studies, review articles, and conference articles were eliminated.

2.4. Extraction of Data. Study data were extracted by two of the authors. Studies that clearly did not meet the inclusion criteria were excluded by reading the title and abstract. After reading the full text, they decided whether to include these studies which may meet the inclusion criteria. If there was a problem of disagreement between the two authors, it was resolved through negotiation or decided after discussion with the third researcher.

2.5. Study Quality. The risk of bias of RCTs was assessed using the Cochrane Collaboration's tool from 7 aspects:

(1) Random sequence generation (selection bias)

(2) Allocation concealment (selection bias)

(3) Blinding of participants and personnel (performance bias)
(4) Blinding of outcome assessment (detection bias)

(5) Incomplete outcome data (attrition bias)

(6) Selective reporting (reporting bias)

(7) Other bias

2.6. Outcome Indicators. In eligible articles, the main indicators extracted were the mean reductions in systolic blood pressure and diastolic blood pressure in the sitting position (msSBP, msDBP), the mean reductions in 24-hour ambulatory SBP (maSBP) and 24-hour ambulatory DBP (maDBP), and the number of participants who achieved BP, msSBP, and msDBP control. The safety outcome was any AEs occurring during the follow-up point.

2.7. Statistical Analysis. This meta-analysis was performed using the statistical software RevMan 5.3. For the continuous variables, such as msSBP, msDBP, maSBP, and maDBP, the data was represented as the weighted mean difference (WMD) between the experimental group and the control group, with a 95\% confidence interval (CI), and we performed subgroup analysis to evaluate the effect about BP control based on different doses of LCZ696. For binary variables, such as AEs, the effect size was represented as an odds ratio (OR) with a 95\% CI. The chi-square test was used to assess heterogeneity between studies. When $P>0.1$ or $I^{2}<50 \%$, the fixed-effect model was applied; otherwise, the random-effect model was used for data analysis.

2.8. Publication Bias. Funnel plots and Egger test were used to assess potential publication bias. When two-sided $P$ value $<0.05$, it means there was publication bias.

2.9. Sensitivity Analysis. We conducted sensitivity analysis. In the RevMan5.3 software, the included studies were removed one by one, and the stability of the results was evaluated by analyzing the changes in the pooled WMD and the pooled OR.

\section{Results}

3.1. Study Characteristics. 1172 relevant studies had been found from the above-mentioned databases. According to the inclusion and exclusion criteria, 8 studies [10-17] were finally included. There were 4313 patients, 1917 patients were randomly assigned to the ARBs group, and 2396 patients were randomly assigned to the LCZ696 group.

Studies population included untreated patients (newly diagnosed patients or those who had not been taking any antihypertensive drug for $\geq 4$ weeks before screening) or treated with antihypertensive drugs for $\geq 4$ weeks before screening, and mean sitting systolic BP (SBP) $\geq 140$ to $<180 \mathrm{~mm} \mathrm{Hg}$ at screening (for untreated patients only) or $\geq 120$ to $\leq 160 \mathrm{~mm} \mathrm{Hg}$ at screening and $\leq 180 \mathrm{~mm} \mathrm{Hg}$ at the end of the washout period (for treated patients only). 


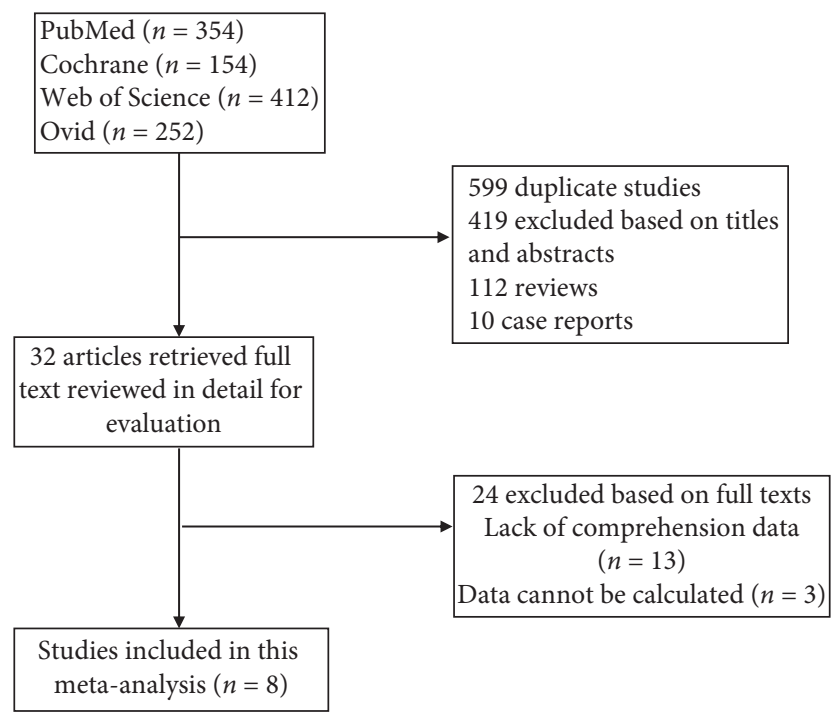

Figure 1: PRISMA flowchart.

Exclusion criteria were patients with severe hypertension, history of angioedema, and previous or current diagnosis of HF (NYHA class II-IV).

The 8 included studies were all clinical RCTs, of which 5 studies compared LCZ696 with olmesartan, and the remaining 3 studies compared LCZ696 with valsartan. The age of patients ranged from 50 to 75 years. Male patients were the majority. The treatment period ranged from 4 weeks to 52 weeks. Some basic characteristics of the included studies are collected in Table 1.

3.2. Study Quality. Quality of studies was assessed by the Cochrane Collaboration's Risk of Bias tool and all included have acceptable quality. All trials were multicenter, randomized, double-blind, active-controlled, parallel-group studies. The included studies were all high-quality articles (level A). Detailed information on the risk of bias is shown in Figure 2.

3.3. Analysis of Blood Pressure Control Results. We mainly included 5 outcome indicators to compare the effect of the drug LCZ696 and ARBs, including msSBP, msDBP, maSBP, maDBP, and blood pressure control rate.

The pooled WMD results showed that LCZ696 had a more pronounced antihypertensive effect compared with the results of ARBs treatment. Indeed, LCZ696 can significantly reduce $\mathrm{msSBP}$ (WMD $-4.29 \mathrm{mmHg}$; $95 \% \mathrm{CI}:-5.37$ to -3.21 ; $P<0.001 ; \quad I^{2}=26 \%$ ) (Figure 3 ), $\operatorname{msDBP} \quad$ (WMD $-1.87 \mathrm{mmHg} ; 95 \% \mathrm{CI}:-2.38$ to $-1.36 ; \quad P<0.001 ; I^{2}=0$ ) (Figure 4), maSBP (WMD $-3.37 \mathrm{mmHg}$; 95\% CI:-4.26 to $-2.47 ; P<0.001 ; I^{2}=59 \%$ ) (Figure 5), and maDBP (WMD $-1.47 \mathrm{mmHg} ; 95 \% \mathrm{CI}:-1.97$ to $\left.-0.97 ; P<0.001 ; I^{2}=40 \%\right)$ (Figure 6).

According to the different doses of LCZ696, we did a subgroup analysis. Taking $200 \mathrm{mg}$ daily of LCZ696 treatment showed a more significant reduction in msSBP (WMD $-4.30 \mathrm{mmHg}$; $95 \% \mathrm{CI}:-6.05$ to $\left.-2.55 ; P<0.001 ; I^{2}=53 \%\right)$,
msDBP (WMD $-1.80 \mathrm{mmHg} ; 95 \% \mathrm{CI}:-2.44$ to -1.15 ; $P<0.001 ; I^{2}=22 \%$ ), maSBP (WMD $-3.44 \mathrm{mmHg}$; 95\% CI: -4.77 to $-2.10 ; P<0.001 ; I^{2}=71 \%$ ), and maDBP (WMD $-1.62 \mathrm{mmHg} ; 95 \% \mathrm{CI}:-2.36$ to $\left.-0.88 ; P<0.001 ; I^{2}=60 \%\right)$. Taking $400 \mathrm{mg}$ daily of LCZ696 treatment also showed a more significant reduction in msSBP (WMD $-4.24 \mathrm{mmHg}$; 95\% CI: -5.59 to $-2.89 ; P<0.001 ; I^{2}=0$ ), msDBP (WMD $-2.00 \mathrm{mmHg} ; 95 \% \mathrm{CI}:-2.84$ to $\left.-1.16 ; P<0.001 ; I^{2}=0\right)$, maSBP (WMD $-3.22 \mathrm{mmHg} ; 95 \% \mathrm{CI}:-4.54$ to -1.90 ; $P<0.001 ; I^{2}=40 \%$ ), and maDBP (WMD $-1.14 \mathrm{mmHg} ; 95 \%$ CI: -1.80 to $-0.48 ; P<0.001 ; I^{2}=0$ )

In addition, LCZ696 had more advantages than ARBs in the control of overall $\mathrm{BP}(\mathrm{OR}=1.78 ; 95 \% \mathrm{CI}: 1.41$ to 2.23 ; $\left.P<0.001 ; I^{2}=0\right)$, msSBP $(\mathrm{OR}=2.02 ; 95 \% \mathrm{CI}: 1.65$ to 2.48 ; $\left.P<0.001 ; I^{2}=0\right)$, and msDBP (OR $=1.71 ; 95 \% \mathrm{CI}: 1.33$ to 2.18; $P<0.001 ; I^{2}=0$ ) (Figure 7).

3.4. AEs. All included studies had reported several drug-related adverse events after treatment with LCZ696 or ARBs [10-17]. A pooled analysis of all adverse events data showed that there were statistical differences between LCZ696 and $\mathrm{ARBs}$ groups in adverse events $(\mathrm{OR}=1.13$; 95\% CI:1.02 to 1.24 ; $\left.P<0.05 ; I^{2}=0\right)$. The results were more favorable to the ARBs group. More exactly, in all the included studies, LCZ696 could increase the incidence of cough in patients $(\mathrm{OR}=1.71 ; 95 \% \mathrm{CI}$ : 1.33 to $\left.2.18 ; P<0.001 ; I^{2}=0\right)$. But except for cough, the results of every adverse event that occurred showed no statistical difference between the LCZ696 and ARBs groups. The detailed analysis of the data is shown in Table 2.

3.5. Publication Bias Review. This meta-analysis only included 8 studies. According to the requirements of the Cochrane Reviewer's Handbook, the funnel chart generally requires more than 10 studies to be meaningful; otherwise, its test power will decrease, and the evaluation of publication bias is not accurate, so publication bias evaluation was not performed. 
TABLE 1: Baseline characteristics of studies incorporated in the meta-analysis.

\begin{tabular}{|c|c|c|c|c|c|c|c|c|c|}
\hline First author & Research design & $E / C$ & $\begin{array}{l}\text { Men, } \\
m(\%)\end{array}$ & Age (y) & $\begin{array}{c}\text { BMI } \\
\left(\mathrm{kg} / \mathrm{m}^{2}\right)\end{array}$ & $\begin{array}{l}\text { Baseline SBP } \\
\quad(\mathrm{mmHg})\end{array}$ & $\begin{array}{l}\text { Baseline } \\
\text { DBP } \\
(\mathrm{mmHg})\end{array}$ & Duration & Number \\
\hline \multirow{2}{*}{$\begin{array}{l}\text { Williams } \\
\text { et al. [10] }\end{array}$} & \multirow{2}{*}{$\begin{array}{l}\text { Multicenter, } \\
\text { randomized, double- } \\
\text { blind, active- } \\
\text { controlled, parallel- } \\
\text { group study }\end{array}$} & $\begin{array}{c}\text { LCZ696 } \\
200 \mathrm{mg}, \mathrm{qd}\end{array}$ & 52 & $68.2 \pm 5.73$ & $28.6 \pm 4.47$ & $160.4 \pm 12.32$ & $85.8 \pm 8.62$ & \multirow[b]{2}{*}{52 weeks } & 229 \\
\hline & & $\begin{array}{l}\text { Olmesartan } \\
20 \mathrm{mg} \text {, qd }\end{array}$ & 52.4 & $67.2 \pm 5.97$ & $29.1 \pm 4.9$ & $160.8 \pm 15.6$ & $85.8 \pm 8.6$ & & 225 \\
\hline \multirow{2}{*}{$\begin{array}{l}\text { Izzo et al. } \\
{[11]}\end{array}$} & \multirow{2}{*}{$\begin{array}{l}\text { Multicenter, } \\
\text { randomized, double- } \\
\text { blind, placebo- and } \\
\text { active-controlled, } \\
\text { parallel-group study }\end{array}$} & $\begin{array}{c}\text { LCZ696 } \\
400 \mathrm{mg}, \mathrm{qd}\end{array}$ & 50 & $61.2 \pm 10.6$ & $29.3 \pm 5.5$ & $159.6 \pm 7.0$ & $90.9 \pm 8.9$ & \multirow[b]{2}{*}{8 weeks } & 142 \\
\hline & & $\begin{array}{l}\text { Valsartan } \\
320 \text { mg, qd }\end{array}$ & 58 & $62 \pm 11.5$ & $30 \pm 5.3$ & $160.0 \pm 7.3$ & $90.2 \pm 9.4$ & & 143 \\
\hline \multirow{2}{*}{$\begin{array}{l}\text { Wang et al. } \\
{[12]}\end{array}$} & \multirow{2}{*}{$\begin{array}{l}\text { Multicenter, } \\
\text { randomized, double- } \\
\text { blind, crossover study }\end{array}$} & $\begin{array}{l}\text { LCZ696 } \\
400 \mathrm{mg} \text { qd }\end{array}$ & 64 & $55.7 \pm 12.5$ & $26.4 \pm 3.8$ & $147 \pm 9.7$ & $147.5 \pm 12.1$ & \multirow{2}{*}{4 weeks } & 36 \\
\hline & & $\begin{array}{l}\text { Valsartan } \\
320 \mathrm{mg} \text {, qd }\end{array}$ & 64 & $58.9 \pm 7.5$ & $25.7 \pm 2.9$ & $90.2 \pm 6.9$ & $90.4 \pm 7.2$ & & 36 \\
\hline \multirow{2}{*}{$\begin{array}{l}\text { Supasyndh } \\
\text { et al. [13] }\end{array}$} & \multirow{4}{*}{$\begin{array}{l}\text { Multicenter, } \\
\text { randomized, double- } \\
\text { blind, active- } \\
\text { controlled, parallel- } \\
\text { group study } \\
\text { Multicenter } \\
\text { randomized, double- } \\
\text { blind, double-dummy, } \\
\text { active-controlled, } \\
\text { parallel group study }\end{array}$} & $\begin{array}{l}\text { LCZ696 } \\
200 \mathrm{mg}, \mathrm{qd}\end{array}$ & 48 & $70.5 \pm 4.67$ & $24.3 \pm 3.15$ & $160.5 \pm 8.41$ & $84.6 \pm 9.74$ & \multirow[b]{2}{*}{14 weeks } & 296 \\
\hline & & $\begin{array}{l}\text { Olmesartan } \\
20 \mathrm{mg} \text {, qd }\end{array}$ & 52.1 & $70.9 \pm 4.67$ & $24.6 \pm 3.24$ & $160.0 \pm 7.99$ & $85.2 \pm 9.83$ & & 292 \\
\hline \multirow{2}{*}{$\begin{array}{l}\text { Schmieder } \\
\text { et al. [14] }\end{array}$} & & $\begin{array}{l}\mathrm{LCZ696} \\
400 \mathrm{mg} \text {, qd }\end{array}$ & 64.9 & $60.5 \pm 7.8$ & $28.1 \pm 4.5$ & $155.3 \pm 9.0$ & $92.7 \pm 8.8$ & \multirow[b]{2}{*}{52 weeks } & 57 \\
\hline & & $\begin{array}{l}\text { Olmesartan } \\
40 \mathrm{mg} \text {, qd }\end{array}$ & 70.2 & $59.2 \pm 13.1$ & $28.6 \pm 3.9$ & $155.0 \pm 9.1$ & $91.7 \pm 8.7$ & & 57 \\
\hline \multirow{3}{*}{$\begin{array}{l}\text { Huo et al. } \\
{[15]}\end{array}$} & \multirow{3}{*}{$\begin{array}{l}\text { Multicenter, } \\
\text { randomized, double- } \\
\text { blind, active- } \\
\text { controlled, parallel- } \\
\text { group study }\end{array}$} & $\begin{array}{c}\text { LCZ696 } \\
200 \mathrm{mg}, \mathrm{qd}\end{array}$ & 52.6 & $57.5 \pm 10.17$ & $26.4 \pm 3.91$ & $158.0 \pm 7.15$ & $90.7 \pm 9.37$ & \multirow{3}{*}{8 weeks } & 479 \\
\hline & & $\begin{array}{l}\text { LCZ696 } \\
400 \mathrm{mg} \text {, qd }\end{array}$ & 51.5 & $58.1 \pm 9.71$ & $26.3 \pm 3.56$ & $157.9 \pm 6.73$ & $89.8 \pm 9.46$ & & 472 \\
\hline & & $\begin{array}{l}\text { Olmesartan } \\
20 \mathrm{mg} \text {, qd }\end{array}$ & 53.9 & $57.4 \pm 10.14$ & $26.4 \pm 3.92$ & $158 \pm 6.53$ & $90.8 \pm 9.57$ & & 484 \\
\hline \multirow{2}{*}{$\begin{array}{l}\text { Cheung } \\
\text { et al. [16] }\end{array}$} & \multirow{3}{*}{$\begin{array}{l}\text { Multicenter, } \\
\text { randomized, double- } \\
\text { blind, active- } \\
\text { controlled, parallel- } \\
\text { group study }\end{array}$} & $\begin{array}{c}\text { LCZ696 } \\
200 \mathrm{mg}, \mathrm{qd}\end{array}$ & 51.6 & $57.1 \pm 10.19$ & $30.5 \pm 5.86$ & $157.1 \pm 9.54$ & $90.4 \pm 10.24$ & \multirow{2}{*}{8 weeks } & 188 \\
\hline & & $\begin{array}{l}\text { Olmesartan } \\
20 \mathrm{mg}, \mathrm{qd}\end{array}$ & 50.8 & $58.0 \pm 9.09$ & $30.6 \pm 5.09$ & $157.8 \pm 10.17$ & $912 \pm 8.89$ & & 187 \\
\hline \multirow{6}{*}{$\begin{array}{l}\text { Ruilope } \\
\text { et al. [17] }\end{array}$} & & $\begin{array}{c}\text { LCZ696 } \\
100 \mathrm{mg}, \mathrm{qd}\end{array}$ & 61 & $53 \pm 10.4$ & N/A & $154.9 \pm 11.89$ & $99.9 \pm 3.62$ & \multirow{6}{*}{8 weeks } & 156 \\
\hline & \multirow{5}{*}{$\begin{array}{l}\text { Multicenter, } \\
\text { randomized, double- } \\
\text { blind, placebo- and } \\
\text { active-controlled, } \\
\text { parallel group, dose } \\
\text { range study }\end{array}$} & $\begin{array}{l}\text { LCZ696 } \\
200 \mathrm{mg} \text {, qd }\end{array}$ & 54 & $54 \pm 9.7$ & \multirow{5}{*}{ N/A } & $156.8 \pm 11.98$ & $99.9 \pm 4.06$ & & 169 \\
\hline & & $\begin{array}{l}\text { LCZ696 } \\
400 \mathrm{mg} \text {, qd }\end{array}$ & 56 & $52 \pm 10.9$ & & $156.3 \pm 12.32$ & $100.4 \pm 4.06$ & & 172 \\
\hline & & $\begin{array}{l}\text { Valsartan } \\
80 \mathrm{mg} \text { qd }\end{array}$ & 58 & $53 \pm 9.6$ & & $154.8 \pm 10.53$ & $99.5 \pm 4.10$ & & 163 \\
\hline & & $\begin{array}{l}\text { Valsartan } \\
160 \mathrm{mg} \text { qd }\end{array}$ & 59 & $53 \pm 9.7$ & & $155.3 \pm 10.79$ & $99.8 \pm 4.41$ & & 166 \\
\hline & & $\begin{array}{l}\text { Valsartan } \\
320 \mathrm{mg} \text { qd }\end{array}$ & 60 & $53 \pm 10.1$ & & $156.0 \pm 11.48$ & $99.5 \pm 3.63$ & & 164 \\
\hline
\end{tabular}

3.6. Sensitivity. Among the 8 studies included in this metaanalysis, different types of studies were eliminated in turn through the analysis of the study characteristics and the differences in patient baseline characteristics; the remaining studies were combined with statistics (pooled WMD or pooled OR) to perform the meta-analysis again. There was no difference between the merged result and the original result.

\section{Discussion}

LCZ696 combines the actions of an ARB and valsartan with a neprilysin inhibitor sacubitril [7]. It is the first new drug known as a dual inhibitory effect.

The main factors and pathogenesis of hypertension are generally believed to be due to the fluid retention and reninangiotensin-aldosterone system (RAAS) excessive 




(a)

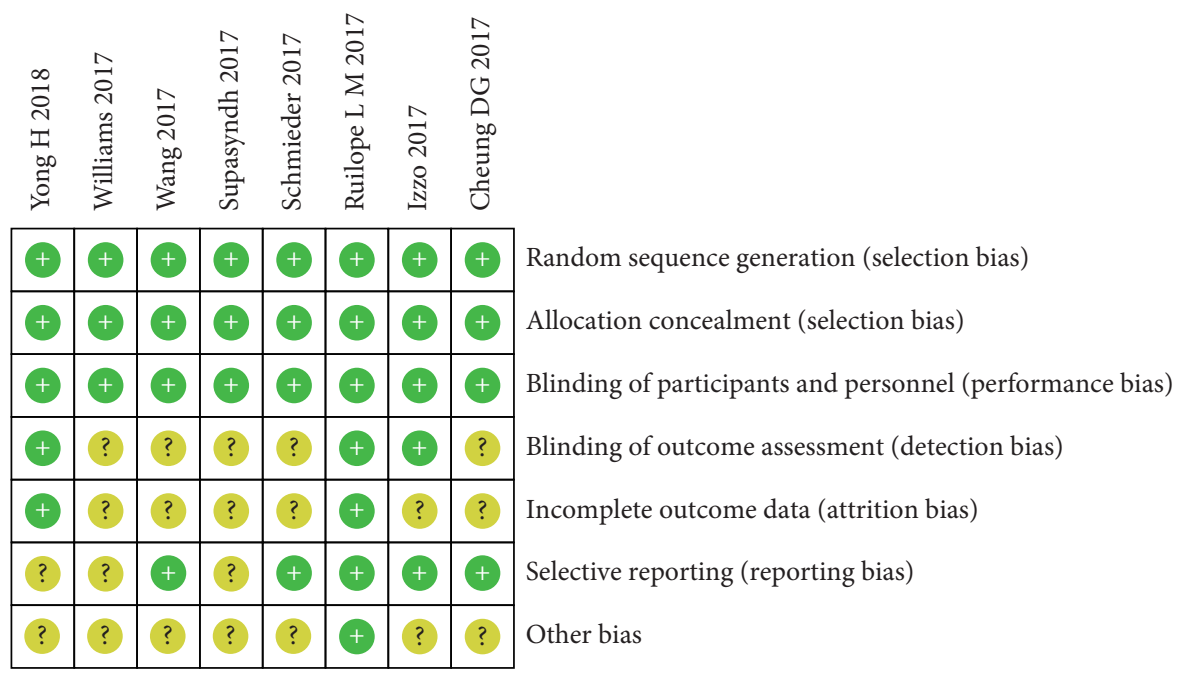

(b)

Figure 2: Risk assessment of bias in clinical studies.

activation. Other studies also had shown that the excessive activation of the sympathetic nervous system (SNS) promotes the reabsorption of the kidneys and damages the pressure natriuretic effect, thereby inducing hypertension [18]. The activation of the RAAS system is caused by many reasons. Angiotensin II promotes smooth muscle contraction, increases ventricular cell synthesis, aldosterone secretion, and arterial smooth muscle cell growth, and can also increase blood pressure. Valsartan inhibits the abnormal activation of the RAAS system by inhibiting angiotensin II receptors, thereby reducing peripheral vascular resistance and reducing fluid retention by inhibiting vasoconstriction and aldosterone secretion. Neprilysin is a neutral endopeptidase that degrades several potentially beneficial vasoactive peptides, including natriuretic peptides, bradykinin, and adrenomedullin, and sacubitril can enhance the effects of these peptides by inhibiting neprilysin, thus promoting vasodilation and increasing urine volume [10]. Therefore, we believe that neprilysin inhibition simultaneously with angiotensin receptor blockade had the potential to counteract some of the mechanisms contributing to arterial stiffening in patients with systolic hypertension and could lower central aortic pressure more effectively than an ARB alone.

An experiment called PARADIGM HF studied by JV McMurray et al. included 8442 patients with HF (NYHA II-IV, LVEF $\leq 40 \%, 88 \%$ ejection fraction less than $35 \%$ ). The therapeutic effect of enalapril on patients with HF was compared with LCZ696. The results showed that LCZ696 can significantly reduce the cardiovascular mortality and HF-related hospitalization rates in patients, and it is better than enalapril in clinical symptoms and long-term prognosis for patients with HF [19]. In addition, LCZ696 can prevent cardiovascular disease and renal insufficiency and has antichronic kidney disease (CKD) and anticardiovascular disease (CVD) effects $[20,21]$. For patients with severe HF, it is necessary to distinguish between advanced HF (AdHF) and end-stage HF. AdHF patients have intolerance to drugs acting on the renal axis, i.e., ACE-I, ARB, or LCZ696, due to hypotension and/or worsening renal function, with risk of $20 \%$ for mortality in 1 year. But end-stage HF patients have an extremely severe cardiomyocyte dysfunction and irreversible organ dysfunction and do not respond to inotropic 


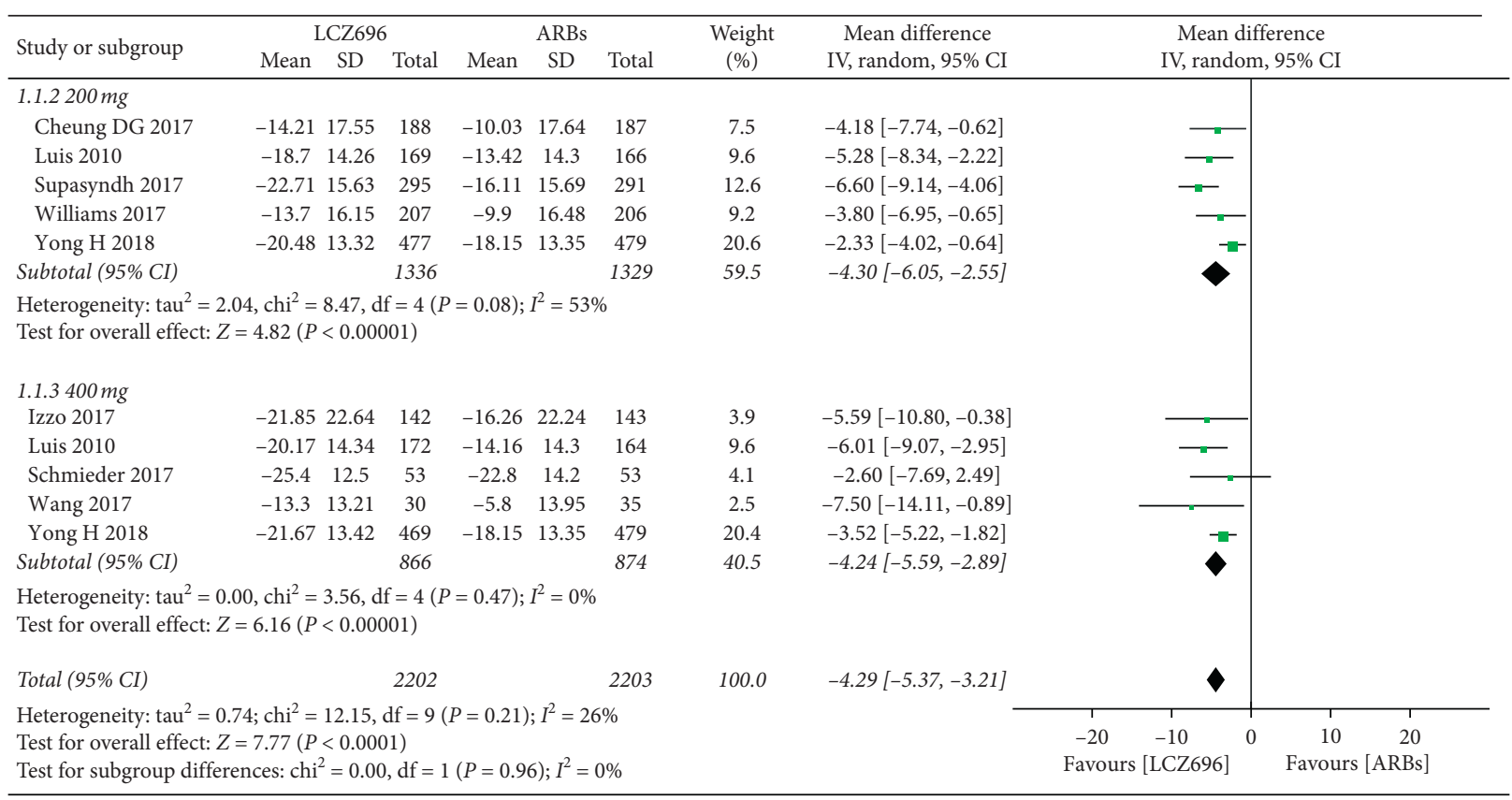

Figure 3: Comparison of LCZ696 with ARBs on mean reduction ( $\mathrm{mm} \mathrm{Hg}$ ) in sitting systolic blood pressure (msSBP).

\begin{tabular}{|c|c|c|c|c|c|c|c|c|c|c|}
\hline \multirow{3}{*}{$\begin{array}{l}\text { Study or subgroup } \\
1.2 .2200 \mathrm{mg}\end{array}$} & \multicolumn{3}{|c|}{ LCZ696 } & \multicolumn{3}{|c|}{ ARBs } & & \multirow{2}{*}{$\begin{array}{l}\text { Mean difference } \\
\text { IV, Fixed, 95\% CI }\end{array}$} & \multirow{2}{*}{\multicolumn{2}{|c|}{$\begin{array}{l}\text { Mean difference } \\
\text { IV, fixed, } 95 \% \text { CI }\end{array}$}} \\
\hline & \multirow[t]{2}{*}{ Mean } & \multirow[t]{2}{*}{$\mathrm{SD}$} & \multirow[t]{2}{*}{ Total } & \multirow{2}{*}{ Mean } & \multicolumn{2}{|c|}{$\begin{array}{c}\text { ARBS } \\
\text { SD }\end{array}$} & & & & \\
\hline & & & & & & \multicolumn{2}{|c|}{ Cotal $\quad(\%)$} & \multirow{2}{*}{$-3.05[-5.00,-1.10]$} & & \\
\hline Cheung DG 2017 & -7.52 & 9.6 & 188 & -4.47 & 9.71 & 187 & 6.8 & & 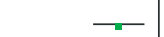 & \\
\hline Luis 2010 & -12.92 & 9.07 & 169 & -9.95 & 9.06 & 166 & 6.9 & $-2.97[-4.91,-1.03]$ & 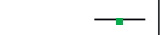 & \\
\hline Supasyndh 2017 & -8.58 & 8.07 & 295 & -6.49 & 8.19 & 291 & 15.1 & $-2.09[-3.41,-0.77]$ & -- & \\
\hline Williams 2017 & -5.9 & 8.81 & 207 & -4.9 & 8.79 & 206 & 9.1 & $-1.00[-2.70,0.70]$ & $-\rightarrow$ & \\
\hline Yong H 2018 & -8.1 & 8.08 & 477 & -6.86 & 8.09 & 479 & 24.9 & $-1.24[-2.27,-0.21]$ & - & \\
\hline Subtotal (95\% CI) & & & 1336 & & & 1329 & 62.8 & $-1.80[-2.44,-1.15]$ & $\diamond$ & \\
\hline \multicolumn{9}{|c|}{$\begin{array}{l}\text { Heterogeneity: } \mathrm{chi}^{2}=5.15, \mathrm{df}=4(P=0.27) ; I^{2}=22 \% \\
\text { Test for overall effect: } Z=5.46(P<0.00001)\end{array}$} & & \\
\hline \multicolumn{11}{|l|}{$1.2 .3400 \mathrm{mg}$} \\
\hline Izzo 2017 & -9.67 & 15.49 & 142 & -7.24 & 13.15 & 143 & 2.3 & $-2.43[-5.77,0.91]$ & . & \\
\hline Luis 2010 & -13.63 & 9.13 & 172 & -10.93 & 9.06 & 166 & 6.9 & $-2.70[-4.64,-0.76]$ & $\longrightarrow$ & \\
\hline Schmieder 2017 & -11.6 & 8.8 & 53 & -11.5 & 8.85 & 53 & 2.3 & $-0.10[-3.46,3.26]$ & - & \\
\hline Wang 2017 & -6.2 & 8.4 & 30 & -4.2 & 9.3 & 35 & 1.4 & $-2.00[-6.30,2.30]$ & - & - \\
\hline Yong H 2018 & -8.8 & 8.23 & 469 & -6.86 & 8.09 & 479 & 24.2 & $-1.94[-2.98,-0.90]$ & - & \\
\hline Subtotal $(95 \%$ CI) & & & 866 & & & 876 & 37.2 & $-2.00[-2.84,-1.16]$ & $\Delta$ & \\
\hline \multicolumn{10}{|c|}{$\begin{array}{l}\text { Heterogeneity: } \text { chi }^{2}=1.81, \mathrm{df}=4(P=0.77) ; I^{2}=0 \% \\
\text { Test for overall effect: } Z=4.68(P<0.00001)\end{array}$} & \\
\hline \multicolumn{7}{|c|}{$\begin{array}{l}\text { Total }(95 \% \mathrm{CI}) \\
\text { Heterogeneity: } \mathrm{chi}^{2}=7.10, \mathrm{df}=9(P=0.63) ; I^{2}=0 \%\end{array}$} & 100.0 & $-1.87[-2.38,-1.36]$ & $\downarrow$ & \\
\hline \multicolumn{7}{|c|}{$\begin{array}{l}\text { Test for overall effect: } Z=7.18(P<0.0001) \\
\text { Test for subgroup differences: } \text { chi }^{2}=0.14, \mathrm{df}=1(P=0.71) ; I^{2}=0 \%\end{array}$} & & \multicolumn{2}{|c|}{$\begin{array}{l}-20 \\
\text { Favours [experimental] }\end{array}$} & $\begin{array}{c}10 \\
\text { Favours [control] }\end{array}$ \\
\hline
\end{tabular}

Figure 4: Comparison of LCZ696 with ARBs on mean reduction ( $\mathrm{mm} \mathrm{Hg}$ ) in sitting diastolic blood pressure (msDBP).

drugs. The presence of the aforementioned features suggests an irreversible condition of end-stage HF, for which drug treatment or advanced therapies, such as ventricular-assist device implantation, are contraindicated and palliative cares should be pursued [22].

Hypertension is a reversible factor of early HF. Longterm hypertension will increase the ejection resistance of the heart and thicken the ventricular wall, which will lead to ventricular remodeling and chronic HF [23]. Hypertension is the most important risk factor for death from cardiovascular and cerebrovascular diseases, bringing a heavy burden of disease to society. In 2017, the number of deaths due to hypertension in China reached 2.54 million, of which about $69 \%$ were deaths from stroke, $54 \%$ were deaths from ischemic heart disease, $41 \%$ were deaths from other cardiovascular diseases, and another $43 \%$ were deaths from chronic kidney disease due to high blood pressure [24]. There are also studies showing that hypertension is a high 


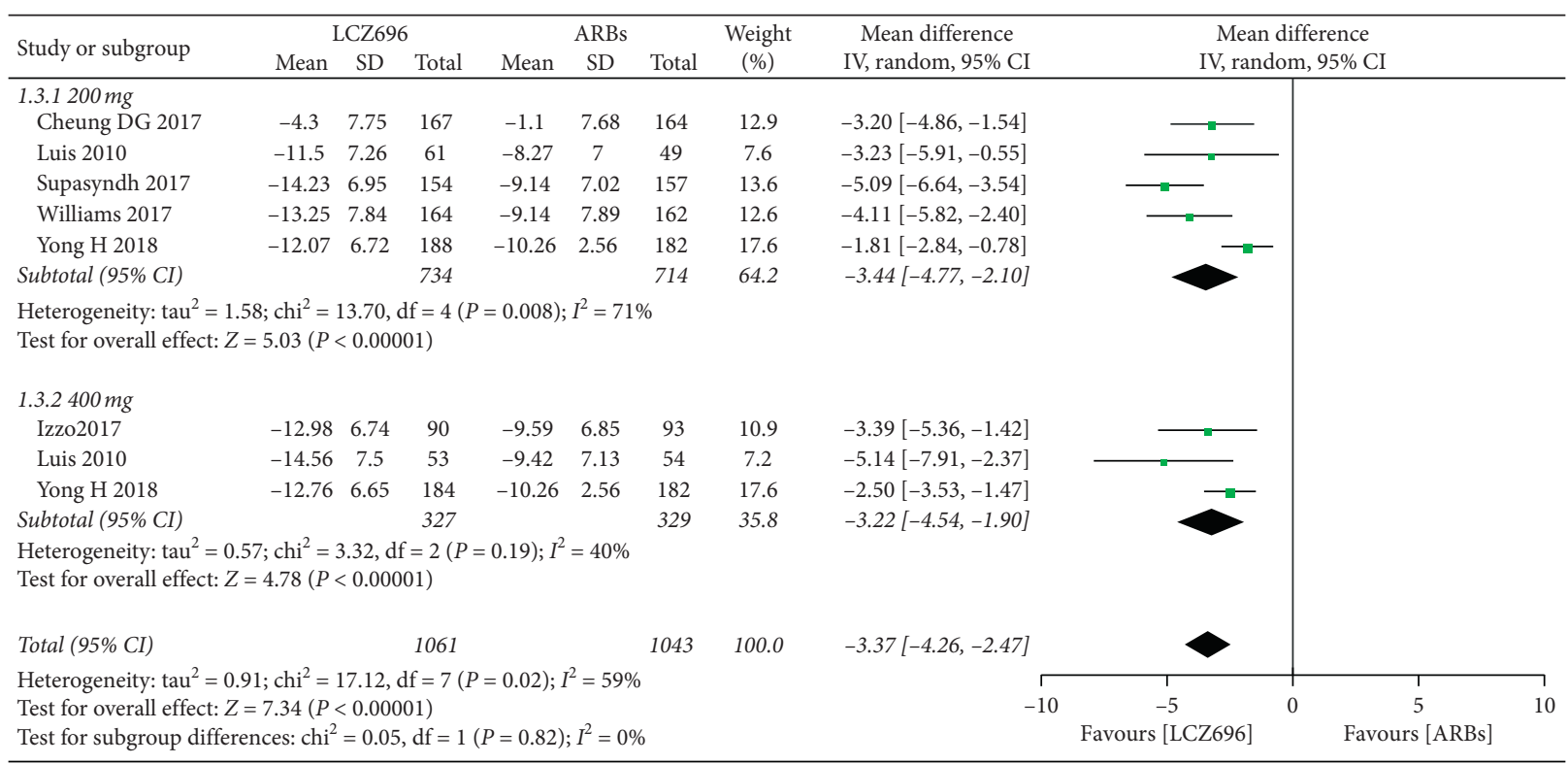

Figure 5: Comparison of LCZ696 with ARBs on mean reduction ( $\mathrm{mm} \mathrm{Hg}$ ) in ambulatory systolic blood pressure (maSBP).

\begin{tabular}{|c|c|c|c|c|c|c|c|c|c|c|}
\hline \multirow{3}{*}{$\begin{array}{l}\text { Study or subgroup } \\
1.4 .1200 \mathrm{mg} \\
\text { Cheung DG } 2017\end{array}$} & \multicolumn{3}{|c|}{ LCZ696 } & \multicolumn{3}{|c|}{ ARBs } & \multirow{3}{*}{$\begin{array}{c}\begin{array}{c}\text { Weight } \\
(\%)\end{array} \\
12.9\end{array}$} & \multirow{3}{*}{$\begin{array}{c}\text { Mean difference } \\
\text { IV, random, } 95 \% \mathrm{CI} \\
-1.90[-2.98,-0.82]\end{array}$} & \multirow{2}{*}{\multicolumn{2}{|c|}{$\begin{array}{l}\text { Mean difference } \\
\text { IV, random, } 95 \% \text { CI }\end{array}$}} \\
\hline & \multirow{2}{*}{$\begin{array}{l}\text { Mean } \\
-2.3\end{array}$} & $\mathrm{SD}$ & Total & Mean & $\mathrm{SD}$ & \multirow[t]{2}{*}{ Total } & & & & \\
\hline & & 5.04 & 167 & -0.4 & 4.99 & & & & & \\
\hline Luis 2010 & -6.78 & 4.5 & 61 & -6.25 & 4.9 & 49 & 6.4 & $-0.53[-2.31,1.25]$ & & \\
\hline Supasyndh 2017 & -6.95 & 3.85 & 154 & -4.47 & 3.88 & 157 & 16.6 & $-2.48[-3.34,-1.62]$ & - & \\
\hline Williams 2017 & -7.44 & 4.74 & 164 & -5.48 & 4.58 & 162 & 14.0 & $-1.96[-2.97,-0.95]$ & & \\
\hline Yong H 2018 & -6.36 & 4.25 & 188 & -5.61 & 4.18 & 182 & 16.6 & $-0.75[-1.61,0.11]$ & & \\
\hline Subtotal (95\% CI) & & & 734 & & & 714 & 66.6 & $-1.62[-2.36,-0.88]$ & & \\
\hline $\begin{array}{l}\text { Heterogeneity: } \mathrm{tau}^{2}= \\
\text { Test for overall effect: }\end{array}$ & $\begin{array}{l}; \mathrm{chi}^{2}= \\
4.29(P\end{array}$ & $\begin{array}{l}9.89, \mathrm{df} \\
<0.000\end{array}$ & $\begin{array}{l}=4(P \\
1)\end{array}$ & $0.04) ; I^{2}$ & $=60 \%$ & & & & & \\
\hline $1.4 .2400 \mathrm{mg}$ & & & & & & & & & & \\
\hline Izzo2017 & -6.2 & 4.17 & 90 & -5.23 & 4.24 & 93 & 11.1 & $-0.97[-2.19,0.25]$ & & - \\
\hline Luis 2010 & -8.32 & 5.17 & 53 & -7.13 & 4.92 & 54 & 5.7 & $-1.19[-3.10,0.72]$ & & - \\
\hline Yong H 2018 & -6.82 & 4.21 & 184 & -5.61 & 4.18 & 182 & 16.6 & $-1.21[-2.07,-0.35]$ & & \\
\hline Subtotal (95\% CI) & & & 327 & & & 329 & 33.4 & $-1.14[-1.80,-0.48]$ & & \\
\hline $\begin{array}{l}\text { Heterogeneity: } \mathrm{tau}^{2}= \\
\text { Test for overall effect: }\end{array}$ & $\begin{array}{l}; \mathrm{chi}^{2}= \\
3.38(P\end{array}$ & $\begin{array}{l}0.10, \mathrm{df} \\
=0.000\end{array}$ & $=2(P$ & $.95) ; I^{2}$ & $=0 \%$ & & & & & \\
\hline Total $(95 \%$ CI) & & & 1061 & & & 1043 & 100.0 & $-1.47[-1.97,-0.97]$ & & \\
\hline $\begin{array}{l}\text { Heterogeneity: } \mathrm{tau}^{2}= \\
\text { Test for overall effect: } \\
\text { Test for subgroup diff }\end{array}$ & $\begin{array}{l}\text {; } \mathrm{chi}^{2}= \\
5.75(P \\
\text { ces: } \mathrm{chi}^{2}\end{array}$ & $\begin{array}{l}11.66, \mathrm{c} \\
<0.000 \\
=0.91\end{array}$ & $\begin{array}{l}\mathrm{f}=7 \\
01) \\
\mathrm{df}=1\end{array}$ & $\begin{array}{l}=0.11) ; 1 \\
=0.34)\end{array}$ & $\begin{array}{l}I^{2}=40^{\circ} \\
I^{2}=0\end{array}$ & & & & $\begin{array}{ll}-4 & -2 \\
\text { Favours [LCZ696] }\end{array}$ & $\begin{array}{cc}0 & 2 \\
& \text { Favours [ARBs] }\end{array}$ \\
\hline
\end{tabular}

FIGURE 6: Comparison of LCZ696 with ARBs on mean reduction ( $\mathrm{mm} \mathrm{Hg}$ ) in ambulatory diastolic blood pressure (maSBP).

risk factor for Alzheimer's disease [25]. As mentioned in Severino P. et al.'s article [26], risk factors, such as hypertension, diabetes, hyperlipidemia, smoking, and inflammation, not only can cause the pathological changes of large blood vessels, but also are pathogenic factors of coronary microvessels. These risk factors can lead to abnormal microvascular function through endothelial cell-dependent and independent mechanisms and eventually lead to ischemic heart disease and heart failure. LCZ696 can effectively lower blood pressure. Therefore, it can improve heart failure caused by microcirculation dysfunction, which is of great significance to patients with abnormal microcirculation function.
The efficacy test results show that LCZ696 has a better antihypertensive effect than the ARB drug valsartan alone [27], mainly because sacubitril and valsartan have a certain synergistic effect; on one hand, it inhibits the RAAS system; on the other hand, it can reduce fluid retention. From the perspective of safety, compared with ARBs, LCZ696 in this study showed no significant difference in safety except for a mild cough (these studies did not report patients who gave up LCZ696 treatment because they could not tolerate cough); that is, compared with ARB drugs alone, LCZ696 has the characteristics of a more significant antihypertensive effect and no obvious safety difference. Based on its remarkable effect and safety, LCZ696 may become one of the 


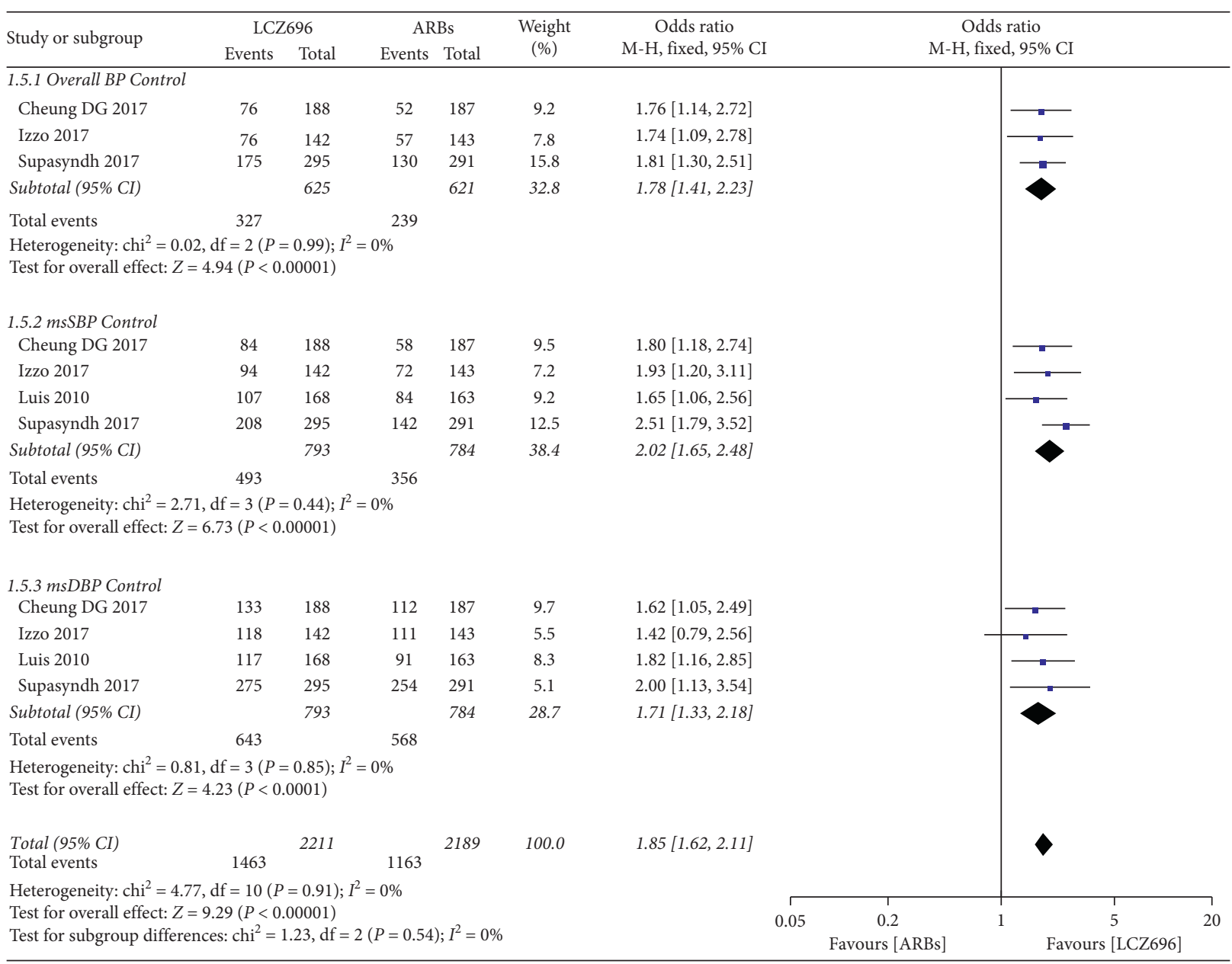

Figure 7: Comparison of LCZ696 with ARBs on BP control.

TABLE 2: Adverse events (AEs) reported in the incorporated studies.

\begin{tabular}{|c|c|c|c|c|c|c|c|c|}
\hline AEs & $\begin{array}{l}\text { No. of studies } \\
(n)\end{array}$ & $\begin{array}{l}\text { LCZ696 group, } \\
n / n\end{array}$ & $\begin{array}{c}\text { ARBs group, } \\
n / n\end{array}$ & Heterogeneity & Analysis model & OR & $95 \%$ CI & $P$ \\
\hline All AEs & 8 & $802 / 2396$ & $612 / 1917$ & $P=0.5, I^{2}=0$ & $\begin{array}{l}\text { Fixed-effect } \\
\text { model }\end{array}$ & 1.13 & $\begin{array}{l}0.99 \\
1.29\end{array}$ & 0.08 \\
\hline $\begin{array}{l}\text { Discontinuations because } \\
\text { of AEs }\end{array}$ & 4 & $29 / 1865$ & $24 / 1389$ & $P=0.58, I^{2}=0$ & $\begin{array}{l}\text { Fixed-effect } \\
\text { model }\end{array}$ & 1.09 & $\begin{array}{l}0.62 \\
1.90\end{array}$ & 0.77 \\
\hline Serious AEs & 5 & $37 / 1721$ & $28 / 1245$ & $P=0.38, I^{2}=5 \%$ & $\begin{array}{l}\text { Fixed-effect } \\
\text { model }\end{array}$ & 1.14 & $\begin{array}{c}0.69 \\
1.88\end{array}$ & 0.6 \\
\hline Dizziness & 8 & $46 / 2396$ & $27 / 1917$ & $\begin{array}{l}P=0.28 \\
I^{2}=18 \%\end{array}$ & $\begin{array}{l}\text { Fixed-effect } \\
\text { model }\end{array}$ & 1.4 & $\begin{array}{l}0.87 \\
2.27\end{array}$ & 0.17 \\
\hline Headache & 7 & $46 / 2100$ & $48 / 1625$ & $P=0.63, I^{2}=0$ & $\begin{array}{l}\text { Fixed-effect } \\
\text { model }\end{array}$ & 0.83 & $\begin{array}{l}0.55 \\
1.24\end{array}$ & 0.36 \\
\hline Diarrhea & 5 & $18 / 1855$ & $13 / 1381$ & $P=0.85, I^{2}=0$ & $\begin{array}{l}\text { Fixed-effect } \\
\text { model }\end{array}$ & 1.3 & $\begin{array}{l}0.65 \\
2.62\end{array}$ & 0.46 \\
\hline Nasopharyngitis & 7 & $67 / 1445$ & $52 / 1433$ & $P=0.84, I^{2}=0$ & $\begin{array}{l}\text { Fixed-effect } \\
\text { model }\end{array}$ & 1.3 & $\begin{array}{c}0.89 \\
1.89\end{array}$ & 0.17 \\
\hline Edema & 3 & $8 / 559$ & $3 / 555$ & $P=0.42, I^{2}=0$ & $\begin{array}{l}\text { Fixed-effect } \\
\text { model }\end{array}$ & 2.25 & $\begin{array}{l}0.69 \\
7.35\end{array}$ & 0.18 \\
\hline $\begin{array}{l}\text { Upper respiratory tract } \\
\text { infection }\end{array}$ & 6 & $44 / 2303$ & $28 / 1824$ & $P=0.50, I^{2}=0$ & $\begin{array}{l}\text { Fixed-effect } \\
\text { model }\end{array}$ & 1.22 & $\begin{array}{l}0.75 \\
1.97\end{array}$ & 0.42 \\
\hline Cough & 6 & $39 / 2043$ & $13 / 1568$ & $\begin{array}{l}P=0.27 \\
I^{2}=22 \%\end{array}$ & $\begin{array}{l}\text { Fixed-effect } \\
\text { model }\end{array}$ & 2.38 & $\begin{array}{l}1.27 \\
4.47\end{array}$ & $0.007^{*}$ \\
\hline
\end{tabular}


TABLE 2: Continued.

\begin{tabular}{|c|c|c|c|c|c|c|c|c|}
\hline AEs & $\begin{array}{l}\text { No. of studies } \\
(n)\end{array}$ & $\begin{array}{l}\text { LCZ696 group, } \\
n / n\end{array}$ & $\begin{array}{c}\text { ARBs group, } \\
n / n\end{array}$ & Heterogeneity & Analysis model & OR & $95 \% \mathrm{CI}$ & $P$ \\
\hline Arthralgia & 4 & $10 / 1425$ & $10 / 953$ & $P=0.40, I^{2}=0$ & $\begin{array}{c}\text { Fixed-effect } \\
\text { model }\end{array}$ & 0.93 & $\begin{array}{l}0.40 \\
2.18\end{array}$ & 0.87 \\
\hline Back pain & 3 & $10 / 428$ & $20 / 425$ & $\begin{array}{l}P=0.17 \\
I^{2}=44 \%\end{array}$ & $\begin{array}{l}\text { Fixed-effect } \\
\text { model }\end{array}$ & 0.49 & $\begin{array}{l}0.23 \\
1.05\end{array}$ & 0.07 \\
\hline Influenza & 3 & $16 / 428$ & $15 / 425$ & $P=0.53, I^{2}=0$ & $\begin{array}{c}\text { Fixed-effect } \\
\text { model }\end{array}$ & 1.07 & $\begin{array}{l}0.52 \\
2.19\end{array}$ & 0.86 \\
\hline Hypotension & 2 & $4 / 417$ & $7 / 412$ & $P=0.47, I^{2}=0$ & $\begin{array}{c}\text { Fixed-effect } \\
\text { model }\end{array}$ & 0.56 & $\begin{array}{l}0.16 \\
1.93\end{array}$ & 0.36 \\
\hline Dyspepsia & 2 & $8 / 639$ & $2 / 636$ & $P=0.98, I^{2}=0$ & $\begin{array}{c}\text { Fixed-effect } \\
\text { model }\end{array}$ & 4.05 & $\begin{array}{l}0.86 \\
19.19\end{array}$ & 0.08 \\
\hline Hyperuricemia & 2 & $38 / 1247$ & $35 / 776$ & $P=0.39, I^{2}=0$ & $\begin{array}{c}\text { Fixed-effect } \\
\text { model }\end{array}$ & 0.71 & $\begin{array}{c}0.44 \\
1.15\end{array}$ & 0.17 \\
\hline Abdominal pain & 2 & $5 / 417$ & $3 / 412$ & $\begin{array}{l}P=0.09 \\
I^{2}=66 \%\end{array}$ & $\begin{array}{l}\text { Random-effect } \\
\text { model }\end{array}$ & 1.19 & $\begin{array}{l}0.05 \\
27.98\end{array}$ & 0.91 \\
\hline
\end{tabular}

first-line drugs for the treatment of hypertension, but large sample experiments are still needed to confirm this conclusion.

This study systematically evaluated all currently included studies on the effect and safety of LCZ696 in blood pressure control. The research results showed that, compared with ARBs, LCZ696 can effectively reduce msSBP, msDBP, maSBP, and maDBP and improve blood pressure control rate. At the same time, compared with ARBs, LCZ696 only increased the incidence of cough, and there were no more adverse events when the dose of LCZ696 was increased. This showed that LCZ696 can benefit more hypertensive patients.

\section{Limitations}

First of all, this study strictly followed the inclusion and exclusion criteria and finally only included 8 studies. The number of studies was relatively small, and a larger research sample size is needed to fully reflect the effectiveness and safety of the drug. Secondly, the length of the included studies was inconsistent, and it lacked sufficient basis for the safety evaluation of long-term using LCZ696 in the treatment of hypertension.

\section{Conclusion}

Although this meta-analysis only included 8 studies and 4313 patients, the results showed that, compared with ARBs, LCZ696 showed better advantages in lowering blood pressure and can be used as an alternative antihypertensive drug.

\section{Conflicts of Interest}

The authors declare that they have no conflicts of interest.

\section{References}

[1] K. T. Mills, J. D. Bundy, T. N. Kelly et al., "Global disparities of hypertension prevalence and control," Circulation, vol. 134, no. 6, pp. 441-450, 2016.
[2] M. J. Sorrentino, "The evolution from hypertension to heart failure," Heart Failure Clinics, vol. 15, no. 4, pp. 447-453, 2019.

[3] P. A. James, S. Oparil, B. L. Carter et al., "2014 evidence-based guideline for the management of high blood pressure in adults," JAMA, vol. 311, no. 5, pp. 507-520, 2014.

[4] K. Kario, J. Zhang, and N. Okino, "LCZ696, a first-in-class Angiotensin receptor-neprilysin inhibitor: the first clinical experience in patients with severe hypertension," The Journal of Clinical Hypertension, vol. 18, no. 4, pp. 308-314, 2016.

[5] M. B. Andersen, U. Simonsen, M. Wehland, J. Pietsch, and D. Grimm, "LCZ696 (Valsartan/Sacubitril)-a possible new treatment for hypertension and heart failure," Basic \& Clinical Pharmacology \& Toxicology, vol. 118, no. 1, pp. 14-22, 2016.

[6] G. Ruiz-Hurtado and L. M. Ruilope, "Advantages of sacubitril/valsartan beyond blood pressure control in arterial hypertension," European Heart Journal, vol. 38, no. 44, pp. 3318-3320, 2017.

[7] J. Gu, A. Noe, P. Chandra et al., "Pharmacokinetics and pharmacodynamics of LCZ696, a novel dual-acting angiotensin receptor-neprilysin inhibitor (ARNi)," The Journal of Clinical Pharmacology, vol. 50, no. 4, pp. 401-414, 2010.

[8] R. De Vecchis, C. Ariano, G. Di Biase, and M. Noutsias, "Sacubitril/valsartan for heart failure with reduced left ventricular ejection fraction," Herz, vol. 44, no. 5, pp. 425-432, 2019.

[9] D. Moher, A. Liberati, J. Tetzlaff, D. G. Altman, and The PRISMA Group, "Preferred reporting items for systematic reviews and meta-analyses: the PRISMA statement," $B M J$, vol. 339, p. b2535, 2009.

[10] B. Williams, J. R. Cockcroft, K. Kario et al., "Effects of sacubitril/valsartan versus olmesartan on central hemodynamics in the elderly with systolic hypertension," Hypertension, vol. 69, no. 3, pp. 411-420, 2017.

[11] J. L. Izzo, D. H. Zappe, Y. Jia et al., "Efficacy and safety of crystalline valsartan/sacubitril (LCZ696) compared to placebo and combinations of free valsartan and sacubitril in patients with systolic hypertension: the RATIO study," J Cardiovasc Pharmacol, 2017.

[12] T.-D. Wang, R.-S. Tan, H.-Y. Lee et al., "Effects of sacubitril/ valsartan (LCZ696) on natriuresis, diuresis, blood pressures, and NT-proBNP in salt-sensitive hypertension," Hypertension, vol. 69, no. 1, pp. 32-41, 2017.

[13] O. Supasyndh, J. a. Wang, K. Hafeez, Y. Zhang, J. Zhang, and H. Rakugi, "Efficacy and safety of sacubitril/valsartan 
(LCZ696) compared with olmesartan in elderly asian patients ( $\geq 65$ Years) with systolic hypertension," American Journal of Hypertension, vol. 30, no. 12, pp. 1163-1169, 2017.

[14] R. E. Schmieder, F. Wagner, M. Mayr et al., "The effect of sacubitril/valsartan compared to olmesartan on cardiovascular remodelling in subjects with essential hypertension: the results of a randomized, double-blind, active-controlled study," European Heart Journal, vol. 38, no. 44, pp. 3308-3317, 2017.

[15] Y. Huo, W. Li, R. Webb et al., "Efficacy and safety of sacubitril/ valsartan compared with olmesartan in Asian patients with essential hypertension: a randomized, double-blind, 8-week study," The Journal of Clinical Hypertension (Greenwich), vol. 21, no. 1, pp. 67-76, 2019.

[16] D. G. Cheung, D. Aizenberg, V. Gorbunov, K. Hafeez, C.-W. Chen, and J. Zhang, "Efficacy and safety of sacubitril/ valsartan in patients with essential hypertension uncontrolled by olmesartan: a randomized, double-blind, 8-week study," The Journal of Clinical Hypertension, vol. 20, no. 1, pp. 150158, 2018.

[17] L. M. Ruilope, A. Dukat, M. Böhm, Y. Lacourcière, J. Gong, and M. P. Lefkowitz, "Blood-pressure reduction with LCZ696, a novel dual-acting inhibitor of the angiotensin II receptor and neprilysin: a randomised, double-blind, placebo-controlled, active comparator study," The Lancet, vol. 375, no. 9722, pp. 1255-1266, 2010.

[18] J. E. Hall, “The kidney, hypertension, and obesity," Hypertension, vol. 41, no. 3, pp. 625-633, 2003.

[19] J. J. V. McMurray, M. Packer, A. S. Desai et al., "Angiotensinneprilysin inhibition versus enalapril in heart failure," New England Journal of Medicine, vol. 371, no. 11, pp. 993-1004, 2014.

[20] G. Boerrigter and J. C. Burnett Jr., "Recent advances in natriuretic peptides in congestive heart failure," Expert Opinion on Investigational Drugs, vol. 13, no. 6, pp. 643-652, 2004.

[21] S. Ito, M. Satoh, Y. Tamaki et al., "Safety and efficacy of LCZ696, a first-in-class angiotensin receptor neprilysin inhibitor, in Japanese patients with hypertension and renal dysfunction," Hypertension Research, vol. 38, no. 4, pp. 269-275, 2015.

[22] P. Severino, P. J. Mather, M. Pucci et al., "Advanced heart failure and end-stage heart failure: does a difference exist," Diagnostics, vol. 9, no. 4, p. 170, 2019 Nov 1.

[23] S. Yandrapalli, M. H. Khan, Y. Rochlani, and W. S. Aronow, "Sacubitril/valsartan in cardiovascular disease: evidence to date and place in therapy," Therapeutic Advances in Cardiovascular Disease, vol. 12, no. 8, pp. 217-231, 2018.

[24] M. Zhou, H. Wang, X. Zeng et al., "Mortality, morbidity, and risk factors in China and its provinces, 1990-2017: a systematic analysis for the Global Burden of Disease Study 2017," The Lancet, vol. 394, no. 10204, pp. 1145-1158, 2019.

[25] C. Iadecola, K. Yaffe, J. Biller et al., "Impact of hypertension on cognitive function: a scientific statement from the American heart association," Hypertension, vol. 68, no. 6, pp. e67-e94, 2016.

[26] P. Severino, A. D’Amato, M. Pucci et al., "Ischemic heart disease pathophysiology paradigms overview: from plaque activation to microvascular dysfunction," International Journal of Molecular Sciences, vol. 21, no. 21, p. 8118, 2020.

[27] C. Orsborne, P. S. Chaggar, S. M. Shaw, and S. G. Williams, "The renin-angiotensin-aldosterone system in heart failure for the non-specialist: the past, the present and the future," Postgraduate Medical Journal, vol. 93, no. 1095, pp. 29-37, 2017. 